\title{
The search for milky way halo substructure WIMP annihilations using the GLAST LAT
}

\author{
Larry Wai \\ Stanford Linear Accelerator Center, Stanford, California \\ representing the GLAST LAT Collaboration \\ Dark Matter and New Physics Working Group
}

\begin{abstract}
The GLAST LAT Collaboration is one among several experimental groups, covering a wide range of approaches, pursuing the search for the nature of dark matter. The GLAST LAT has the unique ability to find new sources of high energy gamma radiation emanating directly from WIMP annihilations in situ in the universe. Using it's wide band spectral and full sky spatial capabilities, the GLAST LAT can form "images" in high energy gamma-rays of dark matter substructures in the gammaray sky. We describe a preliminary feasibility study for indirect detection of milky way dark matter satellites using the GLAST LAT.
\end{abstract}

Key words: GLAST; WIMP; dark matter; indirect detection

Particle dark matter in the form of as yet to be discovered weakly interacting massive particles (WIMPs) can be discovered by direct detection (WIMPnucleus scattering), indirect detection (WIMP pair annihilation), or collider production (WIMP pair production). Direct detection depends upon the local density of WIMP particles, whereas indirect detection depends upon the density (squared) of WIMP particles in regions of the galaxy or beyond. Indirect detection also depends upon WIMPs being either Majorana particles or a mixture of roughly equal numbers of WIMPs and anti-WIMPs. Indirect detection experiments can be categorized by particle type which include neutrinos, antimatter, and gamma-rays (1). Here we focus on how the Gamma Ray Large Area Space Telescope (GLAST) Large Area Telescope (LAT) detector (2) may observe the particle nature of dark matter.

The standard model of cosmology relies on a hierarchical distribution of dark matter which on galactic scales implies a significant number of as yet unobserved dark matter satellites with masses less than approximately $10^{7}$ solar 
masses, the mass scale of the known dark matter dominated low surface brightness dwarf galaxies. We have estimated the number of Milky Way dark matter satellites with $>10^{6}$ solar masses observable by GLAST. The dark matter subhalo distribution was estimated with the semi-analytic method of Taylor \& Babul (3). The dark matter satellite distribution is roughly spherically symmetric about the galactic center and extends well beyond the solar orbit; thus the dark matter satellites are located mostly at high galactic latitudes. We assumed that the dark matter satellites emit gamma rays via the decay of neutral pions. The neutral pions are produced in hadronization of final state quarks, tau leptons, or W/Z bosons produced by pair annihilation of WIMPs. As a case study, we calculated the gamma ray flux for the LSP WIMP (SUSY) for the two benchmark points LCC2 and LCC4 as defined in Baltz, et.al. (4). The background was estimated using the EGRET point source subtracted sky map above $1 \mathrm{GeV}$ from Cillis \& Hartman (5). The significance of the dark matter signal was then estimated to be the number of signal events within the satellite tidal radius (or the PSF 68\% containment radius, whichever was bigger) divided by the square root of the number of background events within the same radius. The resulting number of dark matter satellites with significance of at least some number of sigma is shown in figure 1. For these benchmark SUSY points, we would expect to observe in the range of a few dark matter satellites with 5 years of GLAST data.

In order to distinguish a dark matter source from a more typical astrophysical source, such as a pulsar or molecular cloud, we can make use of the GLAST spatial and spectral resolving capabilities. The typical extent of the observable dark matter satellites is in the range of 1 degree. GLAST should be able to resolve these sources using the WIMP annihilation photons above $1 \mathrm{GeV}$, for which the PSF is approximately $1 / 2$ degree. The WIMP pair annihilation spectrum is extremely hard, much harder than any of the EGRET sources, except possibly the galactic center source. Molecular cloud gamma ray sources will typically have a power law energy distribution, which is distinct from the WIMP annihilation spectrum. In figure 2 we plot the counts spectra for a $100 \mathrm{GeV}$ mass WIMP, high latitude $(b=-31 \mathrm{deg}), 10$-sigma source, plus diffuse background. The diffuse background model consists of the optimized background model from Strong, Moskalenko, Reimer (하) plus the isotropic diffuse background described in Sreekumar et. al. (7). Identification of high latitude GLAST sources with energy spectra consistent with WIMP annihilation would provide a excellent targets for imaging atmospheric Cherenkov telescope (IACT) observations. Precision high statistics observations of WIMP sources can yield information on the nature of the WIMP. For example, the measurement of the $\gamma \gamma$ and/or $\gamma Z^{0}$ line branching fraction can yield information complementary to that obtained with accelerator or direct detection experiments. 


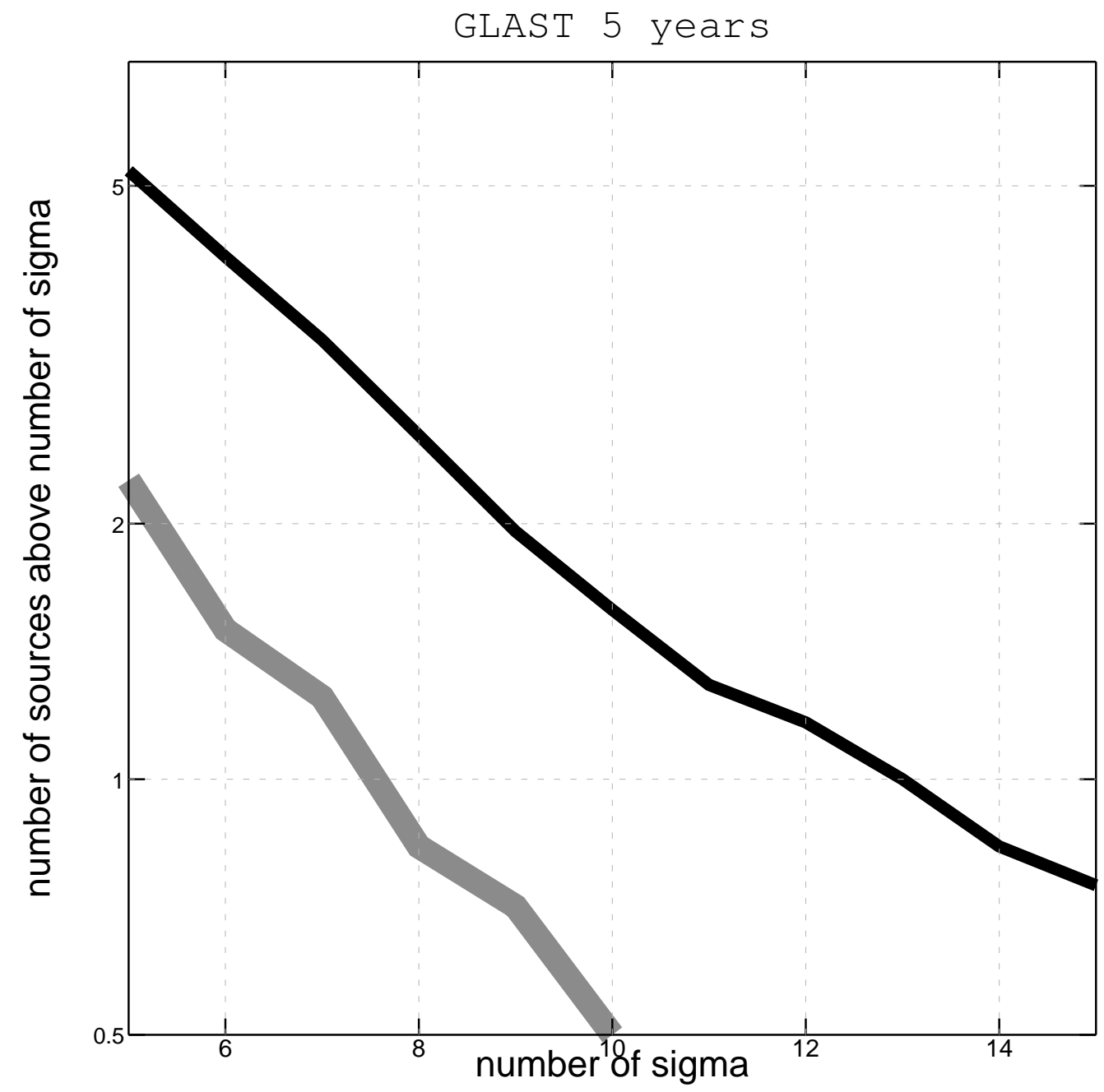

Fig. 1. This graph shows the number of dark matter sources observed in 5-years of GLAST data above a given significance in number of sigma. The dark matter sub-halo distribution for $>10^{6}$ solar masses was estimated with the semi-analytic method of Taylor \& Babul (3). The thin black line corresponds to the SUSY model LCC2 and the thick grey line corresponds to the SUSY model LCC4, as defined in Baltz et.al. (4). The diffuse gamma ray background is estimated using the $>1 \mathrm{GeV}$ source subtracted map from Cillis \& Hartman (5).

\section{References}

[1] J. E. Gunn, B. W. Lee, I. Lerche, D. N. Schramm and G. Steigman, Astrophys. J. 223, 1015 (1978); F. W. Stecker, Astrophys. J. 223, 1032 (1978); J. Silk and M. Srednicki, Phys. Rev. Lett. 53, 624 (1984); J. Silk and H. Bloemen, Astrophys. J. 313, L47 (1987); G. Lake, Nature 346, 39 (1990); L. Bergström, P. Ullio and J. H. Buckley, Astropart. Phys. 9, 137 (1998).

[2] W.B. Atwood et. al., NIM A342, 302 (1994); E.D. Bloom et. al., Space Sci.Rev. 75, 109 (1996); N. Gehrels and P. Michelson, Astropart. Phys. 


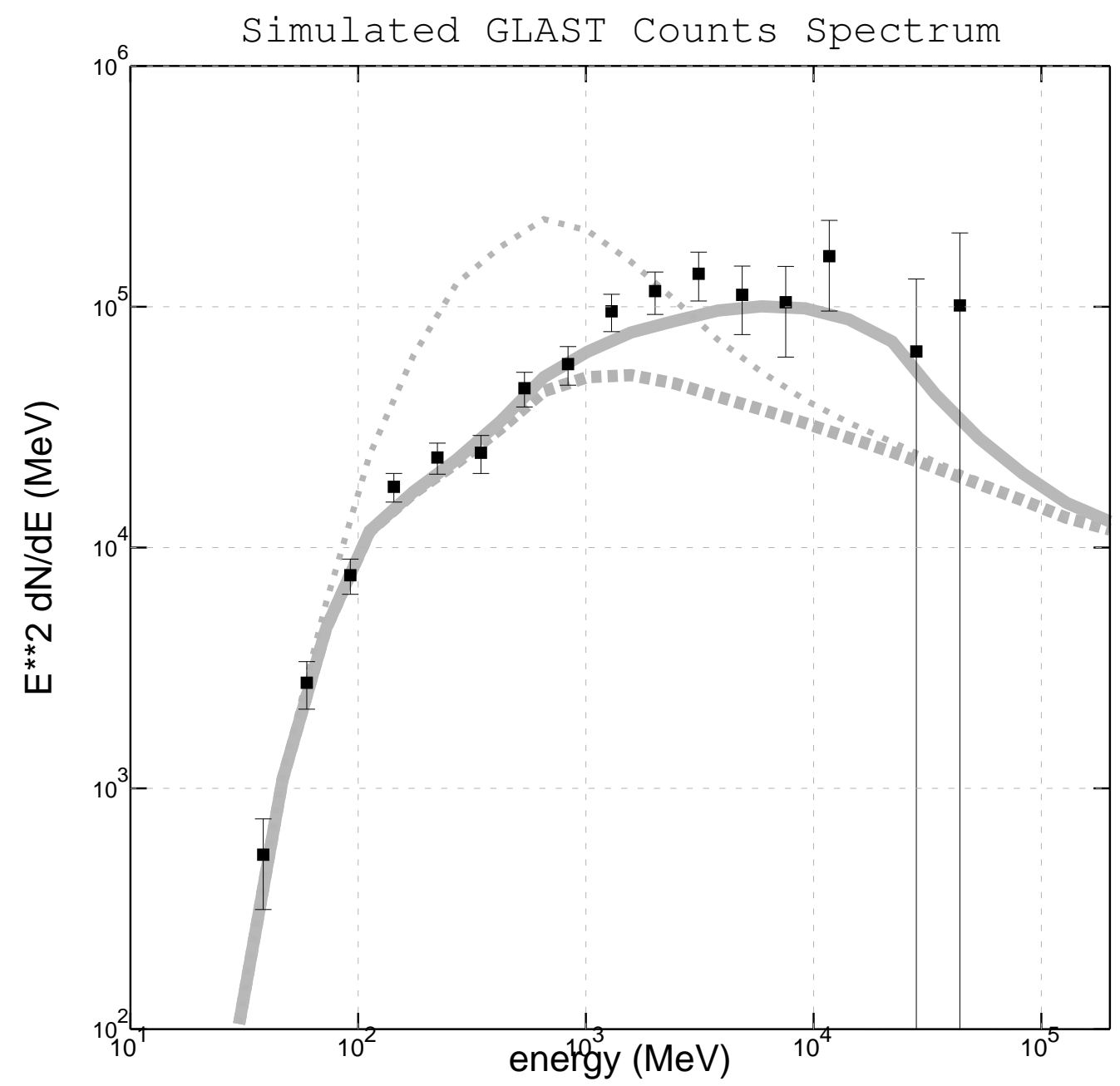

Fig. 2. This graph shows the number of counts versus energy for a simulated 55-days of GLAST. Simulated data from the detector is shown as squares with error bars; this is composed of diffuse background and a $100 \mathrm{GeV}$ mass WIMP (approximately 10-sigma detection). The solid grey line is the fitted counts spectrum, and the thick dotted grey line is the diffuse background only, i.e. optimized background model from Strong, Moskalenko, Reimer (ㅁ) plus an isotropic diffuse background from Sreekumar et. al. (7). The thin dotted grey line is the diffuse background plus a source with a simple powerlaw spectrum, with spectral index -2.6 .

11, 277 (1999)

[3] J. E. Taylor and A. Babul, Mon. Not. R. Astron. Soc. 348, 811 (2004); Mon. Not. R. Astron. Soc. 364, 515 (2005); Mon. Not. R. Astron. Soc. 364, 535 (2005)

[4] E. A. Baltz et al., hep-ph/0602187

[5] A. N. Cillis and R. .C. Hartman, Astrophys. J. 621, 291 (2005)

[6] A. W. Strong, I. V. Moskalenko and O. Reimer, Astrophys. J. 613, 962 (2004); A. W. Strong and I. V. Moskalenko, Astrophys. J. 509, 212 (1998)

[7] P. Sreekumar et al., Astrophys. J. 494, 523 (1998) 